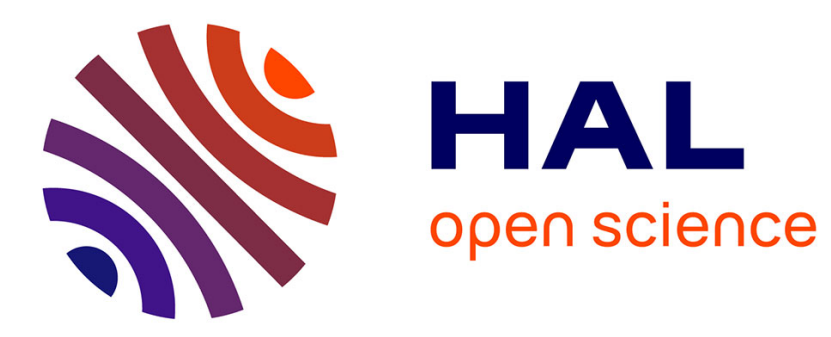

\title{
Terrestres ou volants: les animaux dans la ville antique de Lattes
}

Armelle Gardeisen

\section{To cite this version:}

Armelle Gardeisen. Terrestres ou volants: les animaux dans la ville antique de Lattes. Gallia Archéologie de la France antique, 2008, Lattara/Lattes (Hérault): nouveaux acquis, nouvelles questions sur une ville portuaire protohistorique et romaine, 65, pp.185-192. 10.3406/galia.2008.3341 . halshs-00787481v2

\section{HAL Id: halshs-00787481 https://shs.hal.science/halshs-00787481v2}

Submitted on 19 Dec 2019

HAL is a multi-disciplinary open access archive for the deposit and dissemination of scientific research documents, whether they are published or not. The documents may come from teaching and research institutions in France or abroad, or from public or private research centers.
L'archive ouverte pluridisciplinaire $\mathbf{H A L}$, est destinée au dépôt et à la diffusion de documents scientifiques de niveau recherche, publiés ou non, émanant des établissements d'enseignement et de recherche français ou étrangers, des laboratoires publics ou privés.

\section{(ㅇ)(1) $\$$}

Distributed under a Creative Commons Attribution - NonCommercial - NoDerivatives $\mid 4.0$ 


\title{
TERRESTRES OU VOLANTS : LES ANIMAUX DANS LA VILLE ANTIQUE DE LATTES
}

\author{
Armelle GARDEISEN
}

\begin{abstract}
Mots-clés. Âge du Fer, archéozoologie, animaux de bouche, consommation, déchets culinaires, pratiques bouchères, environnement urbain.

Résumé. Cet article propose une synthèse des études consacrées au domaine animal sur le site de Lattara. Les données acquises sont extraites de leur strict contexte archéologique afin de reprendre, par un examen globalisé de la documentation, un certain nombre de problématiques mettant en jeu les liens qui unissent l'homme à l'animal ainsi que les conséquences de cette relation, souvent inégale, à travers laquelle se dessinent des évolutions techniques et culturelles. Le milieu naturel, l'alimentation, les techniques bouchères, la gestion des déchets, l'environnement animal de la ville, les dépôts animaux ainsi que les matières premières d'origine animale sont les principaux thèmes abordés faisant suite à un historique des recherches archéozoologiques et un inventaire des espèces identifiées.
\end{abstract}

Key-words. Iron Age, archaeozoology, food-bearing animals, consumption, scraps, butchery, urban environment.

Abstract. The results of studies on animals in Lattara are here summarized. Datas are taken from their archaeological context to offer, by a global examination of the documentation, a reassessment of several problems dealing with the relationship between man and animal and consequences of these relations, often unequal, through which technical and cultural evolutions can be drawn. The natural background, food, butchery, scraps management, animal environment in towns, faunal remains and organic raw materials are the main topics being treated after the history of archaeological research and a catalogue of the identified species.

Translation: Isabelle FAUDUET

Schlüsselwörter. Eisenzeit, Archäozoologie, Schlachtvieh, Konsum, Küchenabfälle, Fleischertechniken, städtische Umgebung.

Zusammenfassung. Dieser Abschlussbericht schlägt eine Synthese der Untersuchungen zur Tierwelt in Lattara vor. Die Informationen werden aus ihrem strikt archäologischen Kontext herausgelöst, um sich durch eine weiter gefasste Studie des Fundmaterials mit bestimmten Fragestellungen bezüglich der Beziehung Mensch-Tier, sowie der Konsequenzen dieser oft ungleichen Beziehung zu befassen, die darüber hinaus bestimmte technische und kulturelle Entwicklungen widerspiegelt. Das natürliche Milieu, die Ernährung, die Fleischertechniken, die Abfallwirtschaft, die Lebensbedingungen der Tiere in der Stadt, die Tieropfer sowie die Rohstoffe tierischer Herkunft sind die Hauptthemen, die nach dem geschichtlichen Abriss der archäozoologischen Studien und dem Inventar der identifizierten Arten behandelt werden.

Übersetzung: Isa ODENHARDT-DONVEZ

Cette contribution concerne les recherches consacrées au domaine animal, en particulier celui des animaux terrestres et volants identifiés dans la ville antique de Lattes. Il est intéressant, à l'occasion de cet état des lieux, de proposer à la communauté des archéologues une nouvelle lecture de la documentation traitée au cours des dix dernières années. Ce bilan est donc redevable à l'ensemble des archéozoologues, biologistes et naturalistes qui ont participé aux différents programmes de recherche sur le site.

\section{HISTORIQUE DES RECHERCHES}

Les sources archéozoologiques ont été très tôt intégrées aux problématiques archéologiques grâce à une action thématique de recherche initiale sur les modalités de prélèvement, dont une définition précise était nécessaire à la mise en place de projets d'analyse à long terme, dans la perspective d'une gestion de mobiliers fauniques importants et variés (Colomer, 1989 ; Sternberg, 1989). Un premier bilan 
sur la consommation des animaux d'élevage et de chasse a été donné pour la période allant de la fin du $\mathrm{IV}^{\mathrm{e}} \mathrm{s}$. av. J.-C. au milieu du ${ }^{\text {er }}$ s. apr. J.-C. (Colomer, Gardeisen, 1992). En parallèle, les analyses des restes de poissons étaient intégrées aux études sur l'économie vivrière (Sternberg, 1992) pour ensuite donner lieu à une publication exhaustive (id., 1995) complétée par des analyses ponctuelles répondant aux programmes pluridisciplinaires successifs (id., 1999, 2003 et 2005 ; Piquès, 2005). La malacofaune marine n'a pas été négligée et l'abondance des restes de coquillages a justifié une étude préliminaire consacrée en grande partie à l'alimentation, dans le cadre de la synthèse des données relatives à l'économie vivrière livrée dans la cinquième livraison de Lattara (Brien-Poitevin, 1992). Ces différentes contributions relatives à l'exploitation du milieu marin ou lagunaire ont apporté d'intéressantes informations sur les techniques de pêche ou de collecte, mais aussi sur les préparations de sauces et les modes de conservation (Piquès, 2004) ou encore sur les utilisations secondaires des coquillages (Brien-Poitevin, 1992).

En 1997, la publication du volume Lattara 10, manuel de référence du système d'information archéologique "Syslat », offrait l'occasion d'inclure les données archéozoologiques (mammifères uniquement) sous la forme de fichiers d'inventaire et de traitements statistiques (Py, Gardeisen, 1997) : l'os entrait ainsi à part entière dans la gestion des données archéologiques, au même titre que la céramique ou la stratigraphie par exemple.

Au fur et à mesure de l'évolution des recherches sur la ville, les travaux archéozoologiques se sont naturellement inscrits dans la progression des fouilles et le renouvellement de la programmation scientifique. C'est ainsi que les études se sont placées dans le sillage de la stratégie chronostratigraphique développée sur les zones 1 et 27 qui correspondaient alors aux structures d'habitat visibles du IV ${ }^{\mathrm{e}}$ s. av. J.-C. (Garcia D., 1999 ; Gardeisen, 1999a ; Poitevin, Sénégas, 1999). Ces deux zones présentaient des traits communs dans les couches classiques de remblais : occupation, aménagement ou abandon. En revanche, le dépôt osseux 50004 de la zone 1 s'est distingué par un spectre faunique et par des conditions de dépôt et de conservation originaux (Gardeisen, 1999b). La thématique portant sur l'histoire de la ville au cours du IV $\mathrm{s}$. a été complétée par deux autres contributions : l'une en collaboration avec V. Fabre sur les inhumations d'enfants et les dépôts animaux (Fabre, Gardeisen, 1999), l'autre sur les talus (ou astragales) de caprinés travaillés (Gardeisen, 1999c).

En complément, l'année 2003 a vu l'aboutissement de recherches pluridisciplinaires sur un espace urbain du IV ${ }^{\mathrm{e}}$ s. av. J.-C. la place 123 (Buxó et al. dir., 2003). Cet ouvrage a rassemblé de nombreuses contributions consacrées au monde animal, ainsi que des analyses de sédiments (Cammas, 2003), de parasites (Jouy-Avantin, Moné, 2003) ou de phytolithes (Verdin, 2003) destinées à compléter les approches strictement archéozoologiques (Cheylan, Poitevin, 2003 ; Garcia L., 2003 ; Gardeisen, 2003 ; Geniez, 2003 ; Macé, 2003 ; Sénégas, 2003 ; Sternberg, 2003) autour de la question de l'utilisation de l'espace et des comportements, tant du point de vue anthropique que zoologique.

Enfin les dernières études fauniques réalisées et publiées sont incluses dans la dix-huitième livraison de Lattara (Piquès, Buxó dir., 2005) qui regroupe des approches diverses concernant onze puits gallo-romains, dont trois ont livré de multiples témoins de la présence animale dans la ville (Garcia L., 2005 ; Piquès, 2005 ; Poitevin et al., 2005 ; Poitevin, Cheylan, 2005 ; Ponel et al., 2005 ; Sternberg, 2005 ; Valenzuela Lamas, Gardeisen, 2005 ; Verjat et al., 2005).

\section{LES ANIMAUX TERRESTRES ET VOLANTS DE LATTARA}

La grande diversité des mobiliers fauniques a nécessité une gestion spécifique destinée à faciliter l'accès au matériel pour chacune des disciplines ou spécialités concernées. Ainsi, dès l'arrivée au laboratoire, l'ensemble de la faune a été trié par grandes catégories : macrofaune, microfaune, avifaune, ichtyofaune et conchyliofaune ; les restes de tortues, de mammifères marins, de même que les restes humains, ont été classés individuellement. Au stade de l'analyse, nous tenons compte des deux types de prélèvements : prélèvement à la main, lors de la fouille, et prélèvements au tamisage ou directs (sédiment isolé pour la paléoentomologie). On trouvera ci-après la liste des espèces actuellement identifiées dans ces échantillons.

\section{LES MAMMIFÈRES}

Bos taurus (bovins), Equus caballus, Equus asinus et hybrides (équins), Ovis aries (ovins), Capra hircus (caprins), Sus scrofa (sanglier), Sus domesticus (porcins), Cervus elaphus (cerf), Capreolus capreolus (chevreuil), Oryctolagus cuniculus (lapin), Lepus europaeus (lièvre), Canis familiaris (canins), Canis lupus (loup, identification moléculaire à partir de la pulpe dentaire, voir Tran, 2006), Felis sp. (chat sauvage 
ou domestique), Lynx sp. (lynx), Ursus arctos (ours brun), Lutra lutra (loutre), Meles meles (blaireau), Mustela putorius (putois), Mustela nivalis (belette).

\section{LES MICROMAMMIFÈRES}

Pipistrellus sp. (chauve-souris), Crocidura russula (musaraigne musette), Crocidura suaveolens (musaraigne des jardins), Suncus etruscus (pachyure étrusque), Mus spretus (souris à queue courte), Mus musculus domesticus (souris domestique), Apodemus cf. silvaticus (mulot), Eliomys quercinus (lérot), Microtus agrestis (campagnol agreste), Microtus duodecimcostatus (campagnol souterrain provençal), Microtus cabrerae (campagnol de cabrera), Arvicola sapidus (campagnol amphibie).

\section{LES OISEAUX}

Le site antique de Lattes est caractérisé par une grande diversité ornithologique avec soixante et une espèces parmi lesquelles dominent les anatidés (fuligules surtout, canards, sarcelles, harles, cygnes, oies, bernaches, macreuses) et les galliformes (poule domestique, perdrix) (Garcia L., 1999, 2003 et 2005). On trouve à leur côté de nombreuses autres espèces aquatiques comme le grand cormoran, des ardéidés (butor étoilé, aigrette garzette, héron cendré, héron pourpré, cigogne), des foulques, des grèbes et des grues cendrées, ainsi que des rapaces (milan, vautour moine, buse variable, chouette effraie), des columbidés (pigeon biset, colombin ou ramier, tourterelle des bois), des corvidés (pie, choucas, corbeau, corneille), des grives, des étourneaux et quelques petits passereaux. Enfin, on signale des espèces beaucoup plus rares, voire absentes du milieu local à l'heure actuelle, comme le gypaète barbu et le pélican.

\section{LES REPTILES}

Lacerta lepida (lézard ocellé), Lacerta bilineata (lézard vert), Podarcis sp. ou Psammodromus sp., Elaphe scalaris (couleuvre à échelons).

Tortues : Emys orbicularis (cistude d'Europe) et Mauremys leprosa (émyde lépreuse).

\section{LES BATRACIENS}

Rana sp. (grenouille verte), Pelobates cultripes (pélobate à couteaux), Bufo bufo (crapaud).

\section{LES INSECTES}

De nombreux coléoptères (163 espèces déterminées, voir Ponel et al., 2005) ont été recensés dans les prélèvements des sédiments des puits (Lattara, 18), auxquels étaient associés, dans une moindre mesure, des hyménoptères formicidae (fourmis), des hétéroptères (punaises) ainsi que des diptères (mouches).

\section{LA MALACOFAUNE TERRESTRE}

Trente-trois espèces que nous ne détaillons pas ici se répartissent en deux groupes écologiques principaux (Geniez, 2003) : les espèces méditerranéennes et les espèces hygrophiles (ou semi-forestières). L'unique étude de malacofaune terrestre a été intégrée au programme pluridisciplinaire de la zone 123 (Lattara, 16).

\section{BILANS DES DONNÉES ARCHÉOZOOLOGIQUES}

\section{ÉCOLOGIE ET ENVIRONNEMENT}

Les spectres fauniques déterminés pour les différentes phases de l'occupation de la ville indiquent une exploitation de tous les écosystèmes environnants (plaine, milieux boisés, lagune, étangs, mer) et se démarquent somme toute assez peu de l'environnement faunique actuel ou subactuel. Outre la permanence de quelques mammifères, en particulier les animaux de bouche, il est possible d'observer une certaine évolution dans les rapports chasse-élevage et de mettre en évidence des phénomènes d'apparition-disparition liés à la chronologie et au contexte correspondant.

D'un point de vue environnemental, les animaux chassés traduisent un milieu stable depuis le Bronze final II, voire depuis le Néolithique. Au cours du premier âge du Fer, les cervidés étaient sensiblement aussi nombreux qu'aux périodes précédentes, dans des contextes de pastoralisme actif basé sur les mêmes animaux mais dans des proportions variables d'un site ou d'une époque à l'autre. Il semble que cette situation, observée dans le milieu lagunaire, se retrouve sensiblement sous la même forme dans l'arrièrepays proche ou dans la plaine littorale. Le début du $\mathrm{IV}^{\mathrm{e}} \mathrm{s}$. lattois présente une situation plus contrastée qui favorise le développement des populations de cervidés. Les périodes anciennes ( $\mathrm{V}^{\mathrm{e}}$-IV $\mathrm{e}$ s. av. J.-C.) se caractérisent par un taux d'espèces chassées important qui indique un milieu naturel 
voisin à caractère boisé où évoluent des populations de cervidés (cerfs et chevreuils), des sangliers, mais également des espèces plus marginales comme l'ours, le lynx, ou le lièvre. Ce modèle de prédation connu depuis le Bronze final semble relativement stable jusqu'à la fin $d u \mathrm{IV}^{\mathrm{e}} \mathrm{s}$. Il évolue au $\mathrm{III}^{\mathrm{e}} \mathrm{s}$., époque à partir de laquelle on observe une diminution relative des espèces forestières ainsi que la disparition du lynx, de l'ours et du lièvre, traduisant peut-être plus des choix anthropiques qu'une situation environnementale modifiée. De fait, dans la mesure où cette prédation n'est peut-être pas uniquement le reflet d'un approvisionnement carné de complément, il est délicat de tirer des seuls spectres sauvages de grands mammifères une information strictement paysagère. Au rang des espèces marginales, nous citerons également la loutre, identifiée en zone 52 dans un contexte du III $^{\mathrm{e}} \mathrm{s}$. av. J.-C., les petits mustélidés et le lérot dont la présence plus ou moins régulière est particulièrement marquée au cours des deux premiers siècles de notre ère (en relation avec l'étude des puits gallo-romains, voir Lattara, 18), ainsi que le loup, dont la présence dans un puits gallo-romain de la ville reste à confirmer. Le cortège des micromammifères souligne quant à lui, et sur toute la séquence, le caractère anthropisé des contextes archéologiques avec la présence d'espèces typiquement commensales, comme la souris domestique qui fait son apparition à Lattes et dans le midi méditerranéen au cours $d u \mathrm{IV}^{\mathrm{e}} \mathrm{s}$. : sa présence est ensuite régulière jusqu'au $\mathrm{II}^{\mathrm{e}} \mathrm{s}$. apr. J.-C. La musaraigne musette partage avec la souris, le mulot et le lérot le comportement commensal de ces espèces qui, à l'état naturel, indiquent des milieux humides (pour la souris) et forestiers (pour le lérot). À l'inverse, l'absence du rat noir est notable car on considère généralement qu'il a été introduit en Gaule par les Romains ; néanmoins, on tiendra compte du fait que les données sur cette époque sont peu nombreuses à cause d'une large destruction des niveaux correspondants par les travaux agricoles modernes.

En plus du groupe des espèces anthropophiles, nous remarquons la présence des espèces qui fréquentent les jardins ou les espaces cultivés (musaraigne des jardins, musaraigne étrusque, campagnol agreste et campagnol provençal), celles qui sont inféodées à des milieux particuliers comme les berges des cours d'eau, les canaux, les marais (campagnol amphibie) ou encore les milieux humides composés de prairies alimentées par des sources saisonnières riches en végétation aquatique (tout particulièrement le campagnol de cabrera dont les puits livrent la mention la plus tardive du sud de la France au $\mathrm{I}^{\mathrm{er}}-\mathrm{II}{ }^{\mathrm{e}}$ s. apr. J.-C.). Toujours d'un point de vue biogéographique, nous souli- gnerons la découverte de l'émyde lépreuse dont on sait désormais qu'elle a cohabité avec la cistude dans le delta du Lez au cours des deux premiers siècles de notre ère, fait qui jusqu'alors n'avait jamais été observé. S'il est probable que la présence des micromammifères, outre leur comportement commensal, soit d'ordre naturel (décès sur place, intrusions, pelotes de réjection de chouette effraie), nous pouvons aussi envisager la présence de la cistude comme étant le reflet d'une forme de prédation pratiquée dès le $\mathrm{IV}^{\mathrm{e}} \mathrm{s}$., chasse sans doute à caractère opportuniste ou accidentel, par le piégeage dans les filets de pêche ou les nasses (sans pour autant qu'un ramassage « à la main » ne soit exclu). En revanche, et bien que la consommation directe des cistudes ne soit pas définitivement établie, il semble que l'émyde lépreuse, dont les qualités gustatives ne sont pas reconnues, ait été uniquement piégée. Il n'en reste pas moins que ces deux espèces de tortues traduisent un écosystème fait d'eaux douces, stagnantes ou lentes, riches en plantes aquatiques.

Le milieu aquatique est par ailleurs largement attesté par les spectres ornithologiques composés d'espèces propres aux zones marécageuses méditerranéennes (majoritairement des anatidés) propices à l'accueil d'espèces migratoires (grèbe, cigogne, pélican), mais également par des oiseaux attirés par les activités agricoles (corvidés surtout). Les espèces principalement chassées (fuligule, grèbe, héron cendré, canard, râle, poule d'eau et foulque) proviennent des marais d'eau douce ; la lagune saumâtre n'a que peu de représentants (huîtrier pie, avocette) et les oiseaux de mer se retrouvent en très faibles quantités (fou de Bassan, labbe pomarin, mouette rieuse). En parallèle, l'environnement forestier est traduit par la présence des pigeons, chouettes et hiboux, de même que des espèces plus rares comme le gypaète barbu, le vautour moine, la buse variable, le choucas des tours ou l'outarde ont pu être attirés par les activités anthropiques.

Une autre fenêtre a également été ouverte sur l'environnement proche grâce à l'étude paléoentomologique menée sur deux puits pour la période comprise entre 25 et 125 de notre ère. Le cortège des insectes, essentiellement des coléoptères, suggère des espaces dépourvus d'arbres et dominés par des prairies marécageuses à graminées, interprétation en accord avec les observations archéologiques qui documentent une ouverture de la trame urbaine liée en partie à des aménagements de jardins (confirmé par des couches d'humus significatives) et des modifications architecturales sensibles au cours de la dernière phase d'occupation des secteurs correspondants. 


\section{ÉCONOMIE VIVRIÈRE}

\section{ALIMENTATION GARNÉE}

Les données archéozoologiques montrent que l'élevage constituait, avec la pêche, la cueillette et l'agriculture, l'une des principales activités vivrières des Lattois. Il a été possible de mettre en évidence les grands traits de cet élevage en rendant compte, d'une part, de la variété des produits et, d'autre part, des aspects à la fois traditionnels et spécifiques des activités pastorales et alimentaires au regard de ce qui était pratiqué dans les sites contemporains de l'arrière-pays. L'un des traits majeurs des coutumes alimentaires lattoises est la consommation des porcs que nous avons mise en relation avec les processus d'urbanisation et l'augmentation du nombre des habitants. Bien connu postérieurement dans des cités romaines telles que Nîmes, ce phénomène se met en place plus tôt à Lattes dont le statut de ville portuaire laisse présager de l'introduction précoce de pratiques urbaines qui ne deviennent courantes qu'après la romanisation effective de la région.

Les distributions animales rappellent les associations fauniques observées au cours des III $^{\mathrm{e}}$ et $\mathrm{II}^{\mathrm{e}} \mathrm{s}$. av. J.-C., ce qui laisse penser que le système d'exploitation pastorale est mis en place très tôt dans la cité. Les assemblages de la fin du $\mathrm{V}^{\mathrm{e}} \mathrm{s}$. et de la première moitié $\mathrm{du} \mathrm{IV}^{\mathrm{e}} \mathrm{s}$. témoignent d'une faune abondante et diversifiée qui se caractérise par des taux d'animaux chassés plus importants que dans les périodes suivantes. En effet, nous avons noté un taux d'animaux sauvages au-dessus de la moyenne régionale établie en pourcentage d'individus sur les oppida de l'arrière-pays ainsi que sur les sites de Vaunage entre - 800 et 200 (Columeau, 1978). Les résultats des analyses montrent que s'est maintenue à Lattes une tradition de prédation également mise en évidence dans le Bronze final des sites voisins des Terramares, situés dans un même contexte environnemental de lagunes marécageuses (Columeau, 1985). L'activité cynégétique des Lattois se concentre essentiellement sur les cerfs puis sur les lapins, tableau de chasse qui perdure ensuite et se retrouve encore dans la cité romanisée des deux premiers siècles de notre ère. La présence du sanglier paraît plus anecdotique, même aux débuts de la ville. La finalité de la chasse est clairement déterminée par les observations répétées de marques de boucherie sur les ossements ainsi que par l'exploitation artisanale des bois de chute des cervidés. Il s'agit d'une chasse de subsistance dont le caractère alimentaire est ostéologiquement attesté mais dont les motivations profondes restent mal connues : apport carné complémentaire, destiné peut-être à compenser une baisse de production pastorale (épidémies, guerres, difficultés économiques...) et/ou chasse à caractère culturel dont le rôle social et la valeur symbolique sont ancrés dans des traditions ancestrales ? Il est probable que les habitudes de chasse et de consommation des lapins a généré les tentatives de maintien en captivité de cette espèce prolifique constatées au cours du $\mathrm{I}^{\mathrm{er}}$ s. apr. J.-C. C'est dans ce sens qu'ont été interprétés les assemblages de lagomorphes des puits romains de Lattes, même s'il apparaît que ce protoélevage n’a pas eu de suite, ni localement ni régionalement (Gardeisen, Valenzuela Lamas, 2004). Il n'en reste pas moins que ces stratégies, associées à une augmentation régulière des restes de bovins et à des choix ciblés d'individus immatures dans les populations de porcs ou de caprinés, indiquent une volonté d'obtention de produits de qualité, manifestement toujours disponibles et indispensables à l'approvisionnement d'une ville en développement. En parallèle, il faut évoquer ici la consommation ponctuelle des équidés et des chiens dont les restes osseux sont associés aux déchets de consommation et dont les marques de découpe, lorsqu'elles sont visibles, sont sans équivoques. Nous proposons toutefois une évolution dans ces pratiques, en particulier en ce qui concerne les chiens (Py, 1992b, p. 332). Un comportement différent dans les rejets osseux traduirait, à partir du I ${ }^{\mathrm{er}} \mathrm{s}$. av. J.-C., une tout autre attitude envers les populations canines : en effet, nous ne retrouvons plus dans les assemblages que les éléments a priori non consommables (extrémités des membres) ce qui évoque une utilisation différente (peaux de chiens ?) qui ne soit pas nécessairement alimentaire, tout au moins à l'échelle domestique. Ossements de chiens comme ceux d'équidés portent des marques de découpe, trop rares cependant pour affirmer que tous les individus répertoriés ont été effectivement mangés, mais suffisamment pour les compter dans le rang des animaux consommés.

Enfin, les oiseaux constituent un approvisionnement régulier comme en témoignent les nombreuses traces de découpe ou de préparation (os brûlés) observées sur certaines espèces chassées (canard, fuligule, grèbe, héron, foulque macroule, pigeon et surtout cormoran) ou élevées (poule) pendant les derniers siècles d'occupation de la ville. La chasse au gibier de plume diminue en effet au fur et à mesure que la quantité de poule domestique augmente, et ceci dans le contexte général de retrait de l'activité de prédation.

\section{TECHNIQUES BOUCHÈRES ET PRATIQUES ALIMENTAIRES}

Les traitements des carcasses que l'on déduit des profils de représentation squelettique indiquent une certaine homo- 
généité entre espèces élevées et chassées. Bien entendu, quelques différences apparaissent, en particulier au niveau des régions carpiennes et tarsiennes des cerfs alors que les restes crâniens sont moins nombreux et souvent représentés par des fragments de bois qui ne témoignent pas obligatoirement d'une capture de l'animal. Hormis cela, les représentations des os longs des membres rejoignent celles de la trilogie domestique.

Globalement, les répartitions anatomiques et les traces de découpe, essentiellement bouchères mais également destinées à une manufacture de l'os, témoignent de l'importance des activités liées à l'exploitation des animaux ; tout tend à montrer que ces activités étaient réalisées sur place. Si la localisation des bêtes est délicate à préciser, il est au moins permis d'attester de l'exploitation de carcasses complètes ou quasi complètes localement. Bien que la viande de bœuf soit à la base de l'alimentation carnée des Lattarenses, c'est manifestement le cheptel ovin-caprin qui surpasse en nombre de têtes les autres animaux. On notera la rareté des équidés et une présence discrète des suidés sauvages qui équivaut, en nombre de restes, à celle des cervidés : une situation qui va évoluer dès la fin du IV ${ }^{\mathrm{e}}$ s. av. J.-C. dans le sens de l'augmentation des populations porcines.

Le pourcentage de restes osseux porteurs de marques de découpe varie de $7,30 \%$ à $18,10 \%$ sans évolution notable (les variations sont dues à des contextes et des échantillons différents : dépotoirs, dépôts spécifiques et puits ont été éliminés) avec une moyenne de 7,80\% établie par tranches de cinquante années entre - 450 et notre ère, en fonction $\mathrm{du}$ nombre de restes déterminés $(\mathrm{NRD}$ total $=17$ 257, NR découpe $=1$ 347). Le dépôt 50004, dit de boucherie, présente quant à lui un taux de $21 \%$ de restes osseux striés (Gardeisen, 1999b). La destination alimentaire de l'ensemble des quadrupèdes identifiés justifie l'homogénéité des techniques d'exploitation de leurs carcasses, et donc des pratiques bouchères. Le partage ou la découpe des carcasses, en particulier des animaux de grande taille, répond à cette volonté de mise en pièces préalable à une distribution dont nous ne connaissons pas la portée mais que nous imaginons aisément intra muros. En revanche, des observations répétées de déficits en fémurs d'anatidés ou de foulques laissent supposer un traitement particulier qui suppose la consommation différée de ces morceaux (processus de conservation ou de distribution extra muros). Si l'acte de boucherie et de consommation de la viande est perceptible, les étapes de transformation ou de conservation des produits (graisse, viande, sang) nous échappent, même si tous les éléments domestiques associés à la préparation de la nourriture sont observés in situ: espace de «cuisine ", fours, foyers, grils de cuisson, céramique culinaire, etc.

\section{GESTION DES DÉCHETS}

Les assemblages osseux proviennent de divers contextes archéologiques parmi lesquels les remblais sont les plus nombreux ; viennent ensuite les sols d'habitat (phases d'occupation et d'abandon de maisons ou de pièces), les rues et places, les puits, quelques dépotoirs, des espaces de stockage, la zone portuaire, tous localisés dans l'enceinte de la ville. La première remarque qui s'impose est la propreté des maisons : nous y avons retrouvé de très petits ensembles peu significatifs et toujours épars, composés de fragments osseux ou dentaires de faibles dimensions qui témoignent d'un nettoyage régulier des sols. Cette pratique suppose le rejet des déchets, tout au moins des déchets culinaires, en dehors de l'espace domestique : rues ou places, décharges en dehors de la ville, utilisation d'espaces de dépotoirs spécifiques ou remploi de structures abandonnées (les puits par exemple). Cependant les contextes identifiés comme des zones de décharges restent rares. Nous signalerons à ce titre quelques fosses, les puits, ou encore des espaces extérieurs ouverts. Nous avons en effet constaté que les assemblages osseux provenant des rues étaient souvent caractérisés par des marques d'altérations comme les traces de dents de divers petits charognards qui ont fait office d'éboueurs : les chiens, très probablement, et accessoirement les porcs et des petits mustélidés ou commensaux. De plus, ces espaces devaient également être nettoyés régulièrement, ce qui expliquerait le caractère là encore épars et très fragmenté des assemblages osseux, mais néanmoins quantitativement plus significatifs que ceux issus des maisons. À ce jour, à l'exception des puits, nous n'avons pas identifié de véritables «poubelles » (toutes sortes de déchets confondus). Il est donc probable que, si les déchets étaient momentanément rejetés à l'extérieur des maisons, les aménagements successifs des espaces extérieurs et leur nettoyage aient entraîné l'évacuation des détritus hors de la ville. En effet, même si les puits ont servi de dépotoirs, les assemblages observés à l'intérieur des cuvelages se rapportent plus à des cadavres ou des déchets artisanaux qu'à des déchets domestiques. En parallèle, des squelettes entiers de chiens et de chevaux ont été repérés dans quelques rues, ce qui suppose des cadavres abandonnés et recouverts sans autres précautions. Dans la mesure où les secteurs situés hors les murs n'ont pas été explorés, il est difficile d'estimer quels choix étaient effectués pour l'entretien des espaces publics ou privés. Les études menées 
sur la place 123 permettent néanmoins d'évoquer des accumulations successives de terre et de mobiliers qui présentent des traces de combustion. L'élimination par le feu peut donc être en partie invoquée pour le traitement des déchets mais l'absence de signal osseux (faibles pourcentages d'ossements brûlés quel que soit le type d'assemblage considéré) concernant ces pratiques nous engage à rester prudent à ce sujet.

\section{LES ANIMAUX DANS LA VILLE}

En règle générale, les restes osseux découverts en contexte anthropique nous renseignent sur les animaux ou les portions animales consommés. Le filtre de cette consommation fait que nous reconnaissons des choix alimentaires à partir desquels nous déduisons avec plus ou moins de précision des informations sur la gestion des cheptels et les pratiques pastorales. Ces assemblages osseux évoquent des animaux abattus, des animaux morts dont on n'a que peu de traces au cours de leur vie. Cette remarque s'applique également aux dépôts animaliers. Il n'a pas encore été identifié à Lattes de structures de parcage, d'étables ou d'écuries construites, bien que la présence animale soit effective, comme le démontrent les analyses de micromorphologie (témoins de litières végétales, de piétinement, de parcage) ou de paléoparasitologie (mise en évidence d'œufs de parasites dans des fèces ou des horizons organiques). Certes, le cortège des animaux potentiellement intrusifs ou non consommés par l'homme montre que certaines espèces se sont introduites dans la ville : les espèces commensales, certains microvertébrés, les insectes, les rapaces, ainsi que des individus dont nous avons retrouvé les squelettes entiers ou en connexion d'animaux morts sur place (chiens, équidés, loutre, oiseaux) et ceux qui sont venus se nourrir sur les déchets.

La documentation relative aux animaux vivants dans l'enceinte des villes est de manière générale ténue : elle tient à des traces d'activités (litières, rognages) ou à des assemblages osseux qui ont profité de contextes de conservation optimaux au sein desquels des lots spécifiques ont été reconnus grâce aux contrôles taphonomiques (pelotes de réjection, animaux piégés). Dans le cas de la ville de Lattes, les archéozoologues ont bénéficié du programme d'étude des puits dont la fouille a livré de nombreux restes fauniques, toutes familles animales confondues. Ainsi, les trois puits analysés nous ont offert une occasion rare d'identifier des animaux vivant dans la ville grâce à la reconnaissance de squelettes complets ou quasi complets appartenant à des individus dont les cadavres ont été rejetés. Nous ne sommes pas encore en mesure d'expliquer les causes de ces morts dont l'origine est probablement accidentelle ou pathologique et qui touche les espèces suivantes : bovins, équins, canins, lapins, un jeune suidé, un jeune cervidé. De plus, aucune manipulation ou exploitation des carcasses n'a été observée. Il est donc bien question d'événements ponctuels ou successifs ayant entraîné la mort de nombreux animaux à divers âges. Nous en déduisons que les espèces concernées sont celles qui vivaient dans la ville. Outre les " présents ", il est bon de relever l'absence des ovins, caprins, porcins et volailles domestiques. Si nous pouvons penser que les porcs et les poules évoluaient à proximité des habitations, comme nous l'avons envisagé pour les lapins captifs, il faut admettre que les caprinés étaient parqués hors les murs. La taille et la nature des troupeaux ainsi que l'accès direct aux prairies et aux plaines voisines peuvent expliquer ce fait, de la même manière que les porcs pouvaient également être gardés à l'extérieur. La présence du jeune suidé (peut-être un marcassin) et du faon s'accordent avec l'hypothèse des garennes et de l'ouverture des espaces urbains à des zones de jardins autour du changement d'ère, sur le modèle des jardins d'agrément bien connus dans le monde romain. En revanche, la question de la localisation des bœufs, chiens et chevaux reste en suspens : nous pouvons aisément imaginer la présence de chiens errants (et sans doute régulièrement abattus car trop nombreux), mais cela est plus difficile pour les grands herbivores. À ce stade des recherches, nous nous en tiendrons à l'hypothèse d'animaux isolés ou en petits groupes maintenus dans les cours des maisons.

\section{DÉPÔTS ANIMAUX}

Les animaux sont présents dans la ville de Lattes sous diverses formes : portions carnées consommées, manufacture osseuse, décors (numismatique, chenets, mosaïques, pied de guéridon), intrusions naturelles ou accidentelles, coprolithes, squelettes ou portions squelettiques. Parmi ces derniers, une catégorie particulière de vestiges concerne les dépôts spécifiques trouvés au sein des habitats. Ce type de dépôt, souvent associé à des inhumations d'enfants périnataux, est largement documenté sur le site de Lattes ainsi que sur des sites voisins contemporains (Fabre, 1990 ; Fabre, Gardeisen, 1999). Les principales attestations se répartissent entre le $\mathrm{IV}^{\mathrm{e}} \mathrm{s}$. et le $\mathrm{I}^{\mathrm{er}} \mathrm{s}$. av. J.-C. et présentent un certain nombre de points communs. Leur caractère domestique est déduit de leur situation à l'intérieur des habitations ; ils sont souvent en relation avec un élément architectural ; les animaux associés aux inhumations d'enfants appartiennent 
généralement à des individus juvéniles. Les dépôts animaux sont enfouis en pleine terre, parfois dans des urnes : il s'agit en général de restes crâniens (crânes, mandibules), de portions (pattes d'oiseaux), ou encore de squelettes plus ou moins complets (reptiles, chiots). Les espèces représentées sont des oiseaux, des reptiles, des brebis, des bœufs, des chiots et un porc qui apparaît sous la forme d'un fragment de mandibule associé à un périnatal. Ces pratiques semblent attribuables à une tradition perceptible depuis la fin $d u V^{e} \mathrm{~s}$. qui se perpétue jusqu'au changement d'ère, période à partir de laquelle apparaissent régionalement d'autres modèles de dépôts propres à la civilisation romaine.

\section{MATIÈRES PREMIÈRES ANIMALES}

Outre la consommation ou la préparation des produits carnés, les animaux ont fourni des matières premières et des produits dits secondaires dont l'utilisation est plus ou moins perceptible selon leur potentiel de conservation, leur présence au sein du mobilier archéologique ou encore selon les profils de populations animales établis. En premier lieu, l'os et la corne ont été utilisés comme matière pour l'élaboration de petits objets. Néanmoins, si certains éléments en os ont pu être identifiés directement (Feugère, 1992, p. 303 ; Gardeisen, 1999c ; Raux, 1999, p. 483 et p. 487), le travail de la corne, non conservée, est seulement déduit de l'assemblage de frontaux de bovins et de caprinés qui présentent des marques de découpe de l'étui corné : assemblage spécifique du puits PT471, par exemple (Valenzuela Lamas, Gardeisen, 2005, p. 262). La consommation de lait est quant à elle attestée par les courbes de mortalité des bovins et ovin-caprins ainsi que par la présence de faisselles (Feugère, 1992, p. 294). De même, l'exploitation de la laine est déduite des courbes d'abattage des ovins et caprins et illustrée directement par des restes de tissus (Moulhérat, 2005) et indirectement par la présence de mobiliers liés à l'activité textile, comme les aiguilles et les fusaïoles (Raux, 1999 ; Gardeisen, Raux, 2000). En parallèle, les courbes de mortalité soulignent l'utilisation des animaux, en particulier les bovins et les équins, dans les travaux agricoles : le choix de consommer des animaux de réforme associé à l'observation de quelques pathologies caractéristiques confirment l'utilisation de la force de travail des grands herbivores (traction, portage, etc.).
Parmi les autres produits, nous citerons les fourrures et le cuir dont nous pouvons envisager l'usage à partir des stries de dépouillage observées sur les extrémités distales des membres (Colomer, Gardeisen, 1992 ; Gardeisen, 1999a, b, c et 2003), ainsi que les ailes de rapaces potentiellement exploitées (Garcia L., 1999, p. 637). Enfin, nous pouvons envisager une consommation des œufs, malgré leur absence dans les assemblages issus de l'habitat, à partir des observations qui ont été réalisées dans des dépôts d'offrande à caractère viatique des tombes du second âge du Fer et de la période romaine.

\section{PERSPECTIVES}

Les informations acquises sont donc nombreuses et redevables à l'ensemble des archéologues et archéozoologues qui, par l'analyse de sources très diverses, ont permis de mieux cerner la part du monde animal, terrestre ou volant dans la ville au cours de son histoire. Certes, tous les sujets sont loin d'être épuisés : nous pensons notamment à l'évolution morphologique des populations animales à ce jour peu abordée. Ce volet ne devra pas être négligé car les implications sont fondamentales pour la compréhension de l'évolution des espèces, en particulier des espèces domestiques, mais aussi pour l'évolution des techniques : zootechnie, saillies mulassières, productions spécialisées, etc. À ce jour, les études ont apporté d'importantes informations sur le plan économique et culturel, et ont soulevé des questions d'ordre écologique ou comportemental qui pourront guider les recherches futures. Les programmes à venir devront se concentrer sur ces problématiques, tout en s'attachant à compléter les échantillons osseux des périodes anciennes, concernant le début du fonctionnement de la ville de Lattara, ainsi que les périodes les plus récentes jusqu'à son abandon. Enfin, d'autres problématiques gagneront à être développées en complément des analyses classiques de l'archéozoologie : paléogénétique, alimentation des animaux (phytolithes et microtraces), gestion des cheptels, spatialisation de l'exploitation animale. La richesse du site de Lattes, tout autant que l'effort consenti par les archéologues pour intégrer les études archéozoologiques au processus de fouille, a généré une profusion d'informations qui justifie une mise en perspective des travaux à venir. 